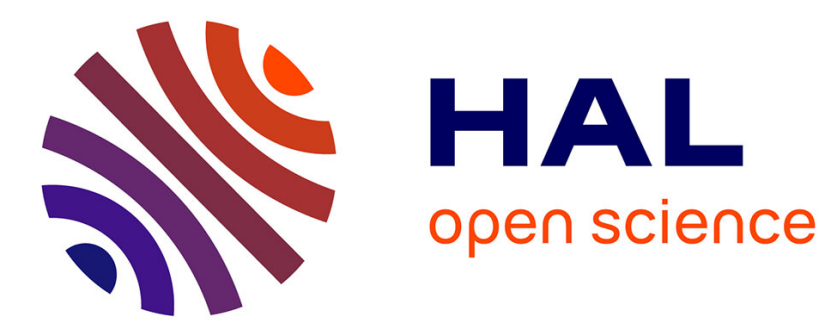

\title{
Helicity cascades in fully developed isotropic turbulence
}

\author{
A. Brissaud, Uriel Frisch, Jacques Léorat, Marcel Lesieur, Alain Mazure
}

\section{To cite this version:}

A. Brissaud, Uriel Frisch, Jacques Léorat, Marcel Lesieur, Alain Mazure. Helicity cascades in fully developed isotropic turbulence. Physics of Fluids, 1973, 16 (8), pp.1366-1367. 10.1063/1.1694520 . hal-02514082

\section{HAL Id: hal-02514082 \\ https://hal.science/hal-02514082}

Submitted on 21 Mar 2020

HAL is a multi-disciplinary open access archive for the deposit and dissemination of scientific research documents, whether they are published or not. The documents may come from teaching and research institutions in France or abroad, or from public or private research centers.
L'archive ouverte pluridisciplinaire HAL, est destinée au dépôt et à la diffusion de documents scientifiques de niveau recherche, publiés ou non, émanant des établissements d'enseignement et de recherche français ou étrangers, des laboratoires publics ou privés. 


\title{
Research Notes
}

Research Notes published in this Section include important research results of a preliminary nature which are of special interest 0 the physics of fluids and new research contributions modifying esults already published in the scientific literature. Research Notes cannot exceed five printed columns in length including space allowed for title, abstract, figures, tables, and references. The abstract should have three printed lines. Authors must shorten galley proofs of Research Notes longer than five printed columns before publication.

\section{Helicity cascades in fully developed isotropic turbulence}

\author{
A. Brissaud \\ Ecole Nationale Superieure de l'Aeronautique et de l'Espace, Toulouse, France \\ U. Frisch \\ Centre National de la Recherche Scientifique, Observatoire de Nice, 06300 Nice, France \\ J. Leorat \\ Observatoire de Meudon, 92190 Meudon, France \\ M. Lesieur \\ Centre National de la Recherche Scientifique, Observatoire de Nice, 06300 Nice, France
}

A. Mazure

Observatoire de Meudon, 92190 Meudon, France

(Received 12 June 1972; final manuscript 7 March 1973)

Based on total helicity conservation in inviscid incompressible flows, the existence of simultaneous energy and helicity cascades is envisaged.

Starting with the work of Steenbeck et al., ${ }^{1}$ the question of isotropic turbulent flows which are not invariant under plane reflections has attracted considerable attention in recent years. Most of this work has focused on the problem of the passive turbulent dynamo, i.e., the generation of large scale mean magnetic fields by prescribed turbulent flows for which $\langle\mathbf{v} \cdot \operatorname{curlv}\rangle \neq 0$; such flows are said to possess net helicity. ${ }^{1,2}$

In this note we shall be concerned with the dynamics of the turbulent flow itself when helicity is present. This fully nonlinear problem has not been given much attention (cf., however, Refs. 3 and 4). Since there is presently no satisfactory theory of fully developed turbulence starting from first principles, we shall examine the influence of helicity from a phenomenological viewpoint.

The classical phenomenological theory uses as a starting point the conservation of the total energy

$$
E=\frac{1}{2} \int v^{2} d x d y d z
$$

for inviscid flows and the assumption that the nonlinear terms in the Navier-Stokes equations give rise to an energy transfer from the large eddies to the smaller ones through a local energy cascade. Under these assumptions, a well-known argument leads to the
Kolmogoroff law for the energy spectrum of isotropic turbulence in the inertial range: $E(k) \sim \epsilon^{2 / 3} k^{-5 / 3}$, where $\epsilon$ is the rate of transfer of energy.

There is, however, another conserved quadratic quantity in inviscid incompressible three-dimensional flows, namely, the total helicity ${ }^{2}$

$$
H=\int \nabla \cdot \operatorname{curl} \nabla d x d y d z \text {. }
$$

Indeed, assuming that the velocity field vanishes at infinity and using the identity

$$
\int \mathbf{A} \cdot \operatorname{curl} \mathbf{B} d x d y d z=\int \mathbf{B} \cdot \operatorname{curl} \mathbf{A} d x d y d \mathbf{z}
$$

valid for vector-fields vanishing at infinity, we obtain

$$
\frac{d H}{d t}=2 \int \mathrm{v} \cdot \frac{\partial}{\partial t} \operatorname{curl} \mathbf{v} d x d y d z .
$$

Then, using the vorticity equation

$$
\frac{\partial}{\partial t} \operatorname{curl} \mathbf{v}=\operatorname{curl}(\mathbf{v} \times \operatorname{curl} \mathbf{v}),
$$

and identity (3), finally, we obtain

$$
\frac{d H}{d t}=2 \int \operatorname{curl} \mathbf{v} \cdot(\mathbf{v} \times \operatorname{curl} \mathbf{v}) d x d y d z=0 .
$$

To the conserved helicity one may associate a 
helicity spectrum $H(k)$, where $H(k) d k$ is the contribution from wavenumbers between $k$ and $k+d k$ to the mean helicity per unit volume $\langle\mathbf{v} \cdot \operatorname{curlv}\rangle . H(k)$, unlike $E(k)$, is a pseudoscalar and not definite positive; however, using the definite-positiveness of the spectral velocity autocorrelation tensor, it may be shown that

$$
|H(k)| \leq k E(k) \text {. }
$$

The situation is somewhat reminiscent of twodimensional turbulence where both the mean energy and mean-square vorticity are conserved; for twodimensional turbulence this leads to two distinct possibilities: an energy cascade and an enstrophy cascade, the energy spectrum following, respectively, a $k^{-5 / 3}$ and a $k^{-3}$ law. ${ }^{5,6}$ It must be stressed, however, that in three dimensions energy can be present without helicity whereas in two dimensions energy and enstrophy are always present simultaneously.

In view of the conservation of helicity, it is interesting to look for the possibility of a helicity cascade in three-dimensional turbulence. If there is a helicity inertial range, where $E(k)$ and $H(k)$ depend only on $k$ and the rate of transfer of helicity $\eta$, the energy and helicity spectra must, for dimensional reasons, take the form ${ }^{7}$

$$
E(k) \sim \eta^{2 / 3} k^{-7 / 3}, \quad H(k) \sim \eta^{2 / 3} k^{-4 / 3},
$$

The possibility of helicity cascades corresponding to local helicity transfer will now be investigated more closely using the simple dynamical argument of Kraichnan. ${ }^{6}$ Let $\Pi(k)$ and $\Sigma(k)$ denote the total rate of energy and helicity transfer from all wavenumbers $<k$ to all wavenumbers $>k$. Following Kraichnan, we assume that $\Pi(k)[\Sigma(k)]$ is proportional to the ratio of the total energy (helicity) $\sim k E(k)[\sim k H(k)]$ available in wavenumbers of the order of $k$ to some effective distortion time $\tau(k)$ of flow structures of scale $k^{-1}$ due to the shearing action of all wavenumbers $\lesssim k$. The distortion time, which is the same for energy and helicity transfer, is given by ${ }^{6}$

$$
\tau(k) \sim\left(\int_{0}^{k} p^{2} E(p) d p\right)^{-1 / 2} .
$$

We therefore write

$$
\Pi(k) \sim k E(k) / \tau(k) ; \quad \Sigma(k) \sim k H(k) / \tau(k) .
$$

We shall say that there is an energy cascade (helicity cascade) if in some "inertial" range, $\Pi(k)[\Sigma(k)]$ is independent of the wavenumber, i.e., $\Pi(k) \equiv \epsilon[\Sigma(k) \equiv \eta]$, where $\epsilon[\eta]$ is the energy [helicity] transfer rate. If we exclude the trivial case $\epsilon=\eta=0$ and the already known case $\boldsymbol{\eta}=0$, two new possibilities remain.

(a) Simultaneous energy and helicity cascades: $\epsilon \neq 0, \eta \neq 0$. Using Eqs. (9) and (10) we obtain the power-law solutions

$$
E(k) \sim \epsilon^{2 / 8} k^{-5 / 8} ; \quad H(k) \sim \eta \epsilon^{-1 / 8} k^{-5 / 8} .
$$

It is easily checked that the dominant contribution to $\tau(k)$ comes from $p \sim k$ in agreement with the idea of localness of both energy and helicity transfer. Notice that solution (11) is compatible with inequality (7) only for large enough $k$.

(b) Pure helicity cascade with no energy cascade: $\epsilon=0, \eta \neq 0 . \Sigma(k)=\eta$ whereas $\Pi(k) \rightarrow 0$ when $k \rightarrow \infty$. Assuming power-law solutions, we obtain a class of solutions

$$
E(k) \sim k^{-n}, \quad H(k) \sim k^{-(5-n) / 2}, \quad \frac{5}{3}<n \leq \frac{7}{8} .
$$

For all solutions, the dominant contributions to $\tau(k)$ still come from $p \sim k$. If we impose the statement that the solution (12) depends only on $\eta$ and $k$, we recover Eqs. (8) corresponding to $n=\frac{7}{8}$.

However, a problem arises with a pure helicity cascade: it appears difficult to inject helicity into the fluid without at the same time injecting some energy. Possibly this difficulty can be overcome, as for twodimensional turbulence, by assuming that energy and helicity are fed into the fluid at a certain wavenumber $k_{i}$; helicity then cascades toward large wavenumbers according to (8) while energy cascades toward small wavenumbers (inverse cascade) according to the usual Kolmogoroff law. In the energy inverse cascade range, $H(k)$ is proportional to $\epsilon^{2 / 3} k^{-2 / 3}$ and $\Sigma(k) \rightarrow 0$ when $k \rightarrow 0$, so there is no helicity transfer.

It may be asked under what conditions solutions of case (b) if they exist, do arise. When the injection of energy and helicity occur at a fixed wavenumber $k_{i}$, a possible answer is that case (b) solutions appear when the helicity injection rate exceeds the critical value which is required by the compatibility of (7) and (11) for $k=k_{i}$, above which stationary solutions of case $a$ cannot exist anymore.

Since all the considerations in this note are purely phenomenological and cannot give conclusive evidence for the existence of helicity cascades, especially of case (b), we intend to test them numerically on a model of turbulence which leads to closed equations for the evolution of $E(k)$ and $H(k)$. As for experimental evidence, we believe that atmospheric turbulence may be a good place to look, because the rotation of the earth combined with the gradients of turbulent intensity produces helicity.

It is a pleasure for us to thank Dr. R. H. Kraichnan for very helpful comments and Professor E. Spiegel for stimulating discussions.

${ }^{1}$ M. Steenbeck, F. Krause, and V. Radler, Z. Naturforsch. A 21, 364 (1966).

${ }^{2}$ H. K. Moffat, J. Fluid Mech. 35, 117 (1969).

${ }^{3}$ S. I. Vainshtein, Zh. Eksp. Teor. Fiz. 61, 612 (1971) [Sov. Phys.-JETP 34, 327 (1972)].

${ }^{4} \mathrm{~A}$. Mazure, Thesis, Observatoire de Meudon, 1972.

${ }^{5}$ R. H. Kraichnan, Phys. Fluids 10, 1417 (1967).

${ }^{6}$ R. H. Kraichnan, J. Fluid Mech. 47, 525 (1971).

${ }^{7}$ M. Lesieur, U. Frisch, and A. Brissaud, Ann. Geophys. 27, 151 (1971). 